\title{
Simulations of combined Neutral Beam Injection and Ion Cyclotron heating with the TORIC-SSFPQL package
}

\author{
R. Bilato ${ }^{1}$, M. Brambilla ${ }^{1}$, O. Maj $^{1,2}$, L.D. Horton ${ }^{3,4}$, \\ C.F. Maggi ${ }^{1}$, J. Stober ${ }^{1}$ \\ ${ }^{1}$ Max-Planck Institut für Plasmaphysik - EURATOM Association - Garching, \\ Germany \\ 2 Max-Planck-Institut für Sonnensystemforschung, Katlenburg-Lindau, Germany. \\ ${ }^{3}$ EFDA-CSU Culham, Culham Science Centre, Abingdon, Oxon OX14 3DB, U.K. \\ ${ }^{4}$ European Commission, B-1049 Brussels, Belgium \\ E-mail: roberto(dot)bilato (at) ipp (dot)mpg (dot) de
}

\begin{abstract}
.
A source describing the injection of fast ions due to the ionization of high-energy neutral beams has been added to the surface-averaged quasilinear Fokker-Planck code SSFPQL (M. Brambilla 1994 Nuclear Fusion 34 1121). For this purpose, the multiplebeam NBI code SINBAD (Y. Feng et al. 1995, Comp. Phys. Comm. 88 161) has been included as a module in SSFPQL, with the modifications required to handle arbitrary axisymmetric equilibria. Alternatively, the Neutral Beam Injection (NBI) source can be built using the output of a Monte Carlo NBI code. We have also added a term describing losses of fast ions during thermalization, and a subroutine evaluating the neutron production rate by nuclear reactions. With these extensions, iterations between SSFPQL and the full-wave solver TORIC (M. Brambilla 1999 Plasma Phys. Control. Fusion 41 1) can now be used to investigate the strong interplay between NBI and Ion Cyclotron (IC) heating.

By comparing the predicted and measured neutron production rates from D-D reactions in a discharge with combined NBI and IC heating in ASDEX Upgrade we obtain a plausible estimate of the importance of fast ion losses, even if their cause cannot be identified. We find, however, that the plasma composition, in particular the presence low Z impurities, plays a more critical role than fast ion losses in limiting the efficiency of this heating scheme.
\end{abstract}

PACS numbers: 52.35.Hr, 52.50.Qt, 52.55.Fa, 52.65.-y, 52.50.Gj

Submitted to: Nuclear Fusion 


\section{Introduction}

Neutral beam injection (NBI) and high frequency (hf) heating with the fast wave (FW) in the ion cyclotron (IC) range of frequencies are standard methods of ion heating in magnetically confined plasmas [1,2]. Simultaneous application of NBI and IC heating can very efficiently produce high-energy ion tails $[3,4,5,6,7]$. In particular, in a plasma in which NBI is sustaining an important fast ion population, strong synergetic effects can be expected with ion heating at IC harmonics $\left(\omega=n \Omega_{\text {ci }}\right.$ with $\left.n \geq 2\right)$, which is a finite Larmor radius (FLR) effect, and, therefore, preferentially accelerates ions with large perpendicular energy, $k_{\perp} \rho_{i} \gtrsim 1$ (here $k_{\perp}$ is the perpendicular wave number and $\rho_{i}=v_{\perp} / \Omega_{\mathrm{ci}}$ the particle Larmor radius). This heating combination has been proposed to boost NBI driven current [8], and has been used to address the physics of fast ions in large tokamaks [9].

Because of the close interplay between fast-ion tails formation and absorption of IC waves, numerical simulations of IC heating in presence of NBI require an iteration loop between a wave solver and a solver of the kinetic Fokker-Planck (FP) equation [10]. The first attempts to quantify ICRF-NBI synergies used simplified models, such as a FP equation averaged over the velocity pitch-angle, and heuristic ICRF power deposition profiles $[8,11,12,13]$. As the level of sophistication of the FP and wave solvers increased $[14,15,16]$, the first attempts of selfconsistent modeling were made [10]. Nowadays, several toroidal full-wave codes have been interfaced with two-dimensional velocity-space Fokker-Planck solvers in order to take into account non-Maxwellian distribution functions in the wave propagation and absorption [17, 18, 19, 20, 21, 22]. Here we present an extension of the FP quasilinear solver SSFPQL, coupled to the full-wave solver TORIC [23], to include in the kinetic equation a source describing NBI and an heuristic model of losses of energetic ions. Compared with other successful approaches to the same problem, such as CQL3D-AORSA [17], GNET-TASK/WM [18], ORBIT-AORSA [21], and VENUS-LEMan [22], the SSFPQL-TORIC package requires relatively modest computing resources, without sacrificing any important aspect of the physics involved. Thus, with the help of scripts automatically driving the iterations, fully converged simulations with very high resolution in real and velocity space can be achieved in a few hours on a laptop. We are aware, however, that execution speed is obtained with simplifications (surface averaging and omission of some toroidal effects on the motion of fast ions) which, while well justified for the self-consistent evaluation of wave propagation and absorption in the IC range of frequencies, are not entirely adequate for addressing, for example, resonant interactions of the most energetic ions accelerated by ICRF with MHD modes [24]. The best approach for this purpose is an orbit-following Monte Carlo code [25, 18, 21] which takes into account the correct Doppler shift in ICRF Monte-Carlo operators [26, 22]. Because of the inherent noise, however, the output of such a code is considerably more difficult to be used as input in a wave solver, although this has been recently attempted [21, 22]. It should be clear, 
on the other hand, that IC waves propagation and absorption depend essentially on the relatively large populations of medium-energy ions for which surface averaging is to a good extent justified, rather than on the interesting but exponentially few ions on really large orbits.

As a preliminary, we briefly recall the main features of TORIC and SSFPQL, referring to $[20]$ for a more detailed description of the two codes and of the interface which has been developed to facilitate the iteration between the two solvers.

TORIC [23, 27] solves Maxwell's equations in axisymmetric toroidal plasmas, assuming a constitutive relation (relation between high-frequency field and high-frequency plasma current) obtained from the linearized Vlasov equation by expanding the wave field in toroidal and poloidal Fourier components. The model includes propagation and damping of externally launched fast waves (FW), as well as of Ion Bernstein (IBW) and Ion Cyclotron (ICW) waves excited by linear mode conversion (LMC) near ion-ion resonances. The absorption channels are fundamental and first harmonic IC heating of ions, and Landau and Transit Time damping of electrons. Optionally, damping of the FW at higher IC harmonics can be taken into account [28]. The coefficients of the wave equations are evaluated for arbitrary distribution functions using the information transferred from SSFPQL [20]. Recent applications of the TORIC code can be found in $[29]$.

The Fokker-Planck quasilinear solver SSFPQL [30] evaluates the steady-state quasilinear distribution function of ions heated at the fundamental and first harmonic IC resonance by balancing the surface-averaged quasilinear operator (QLO) with the linearized collision operator describing collisions with a Maxwellian background plasma. The solution is obtained as a truncated series in Legendre polynomials. A special Bessel function identity is used to guarantee that the truncated Legendre expansion of the QLO remains positive definite up to sufficiently high energies. As discussed in [20], this approach has some limitations, most of them common to all kinetic equations based on surface averaging. On the other hand it is very fast, and allows to obtain the quasilinear distribution functions (QLDF) on a dense radial mesh, as required for iteration with the wave solver. A very efficient interface between the two codes evaluates the coefficients of the wave equations using the QLDF provided by SSFPQL, in particular the singular integrals in the reactive part of these coefficients, at all points of the radial mesh, and transmits the information to TORIC.

In section 2 we describe how NBI sources are included in SSFPQL. This requires an interface with a code which evaluates the distribution of nascent ions as the neutral beams penetrate into the plasma. The information can be obtained as intermediate output of the Monte Carlo code FAFNER [31]. This output, however, is very noisy, and information is lost in the smoothing process required to use it in a kinetic code like 
SSFPQL. As a more convenient alternative, the multiple-beam NBI code SINBAD [32] has been extended to accept arbitrary numerical tokamak equilibria, and inserted as a module in SSFPQL.

A few further extensions to the SSFPQL code have been made to facilitate the investigation of the suprathermal ion populations produced by IC and/or NBI heating. When the fast ions are reactants of nuclear reaction with neutron yield, the effectiveness of the fast-ion tail formation can be estimated from the enhancement of the neutron rate [14]. To make possible the comparison with experimentally measured neutron rates during discharges with NBI and/or IC heating, a synthetic diagnostics has been implemented, based on the efficient algorithm due to Cordey et al. [33] for the evaluation of the reactivity $\langle\sigma v\rangle$ when the distribution functions are expanded in Legendre polynomials (section 3 ).

As already noted in [20], an important limitation of SSFPQL has been the absence of a term describing losses of energetic ions during slowing down. Comparisons of the numerical and experimental neutron rates in the presence of simultaneous NBI and harmonic IC heating have further emphasized the need of such a term. A realistic model of how fast ion losses (FIL) depend on energy, pitch angle, radial position, and other parameters, is not available, but several heuristic loss models have been proposed $[13,34,35]$. The FIL loss model which has been incorporated in SSFPQL is presented in section 4 .

Finally, in Section 5 we present a few applications, discussing the behaviour of the iteration between the two codes, and the role of FIL losses and impurities to reproduce the measured rates of neutron production in ASDEX Upgrade. Although of limited scope, this example shows that our numerical tools would be adequate to analyze a more extended database of discharges with simultaneous NBI and IC heating when available. The conclusions are summarized in section 6 .

\section{The Neutral Beam Injection source}

With simultaneous NBI and RF heating the steady-state quasilinear surface-averaged kinetic equation for ion species $i$ solved by SSFPQL is

$$
0=\left(\frac{\partial F_{i}}{\partial t}\right)_{\text {coll }}+\left(\frac{\partial F_{i}}{\partial t}\right)_{\mathrm{QL}}+\mathcal{S}_{i}^{\Delta \mathrm{T}}(\psi, v)+\mathcal{S}_{i}^{\mathrm{nbi}}(\psi, v, \mu)+\mathcal{L}_{i}^{\mathrm{FIL}}(\psi, v, \mu)
$$

Here $\psi$ labels magnetic surfaces, $v$ is the particle velocity (in SSFPQL $v$ is normalized to the thermal speed $v_{t h i}=\left(2 T_{i} / m_{i}\right)^{1 / 2}$ of the background ions), and $\mu$ the velocity pitch angle $v_{\|} / v$. The first three terms are respectively the linearized collision operator, the IC quasilinear operator, and the source term required to maintain in steady-state charged species at different temperatures; these terms are discussed in [20]. New are 
the source $\mathcal{S}_{i}^{\text {nbi }}$, representing NBI, and the loss term $\mathcal{L}_{i}^{\mathrm{FIL}}$ which describes losses of fast ions during slowing down.

The source describing NBI is written

$$
\mathcal{S}_{i}^{\mathrm{nbi}}(\psi ; v, \mu)=\sum_{k=1}^{N_{s}} \frac{\delta\left(v-v_{i, k}\right)}{v_{i, k}^{2}} \frac{S_{i, k}(\psi, \mu)}{2 \pi}-\mathcal{L}_{i}^{\mathrm{nbi}}
$$

where the summation extends over the injection energies $E_{i, k}=m_{i} v_{i, k}^{2} / 2$ of species $i$. The term $\mathcal{L}_{i}^{\text {nbi }}$ describing losses of thermal particles has been added to compensate the NBI ionization rate so that a steady-state is possible. Since the energy dispersion of the beams around the mean values $E_{i, k}$ is extremely narrow, the velocity dependence of $\mathcal{S}_{i}^{\text {nbi }}(\psi ; v, \mu)$ is well described by Dirac-delta functions. While the multiple-beam code SINBAD directly evaluates the quantities $S_{i, k}(\psi, \mu)$ at the points of the desired mesh in velocity and pitch angle, a special interface had to be developed to obtain them from the output of the Monte Carlo code FAFNER [31].

In principle, the evaluation of $\mathcal{S}_{i}^{\text {nbi }}$ from the results of a Monte Carlo code should proceed as follows. Let $\Delta E$ be a range of energy sufficiently small to separate well the different energy levels in the beams. On a mesh in $\psi$ with step $\Delta \psi$, and in $\mu$ with step $\Delta \mu$

$$
2 \pi \mathcal{S}_{i, k}\left(\psi_{r}, \mu_{s}\right)=\sum_{p \in D_{r, s}} \frac{w_{p}}{\mathcal{V}\left(\psi_{r}\right) \Delta \psi \Delta \mu}
$$

where the sum extends to all markers $w_{p}$ of the Monte Carlo simulation lying in the domain $D_{r, s}$ defined by $E_{i, k}-\Delta E / 2 \leq E \leq E_{i, k}+\Delta E / 2, \psi_{r}-\Delta \psi / 2 \leq \psi \leq \psi_{r}+\Delta \psi / 2$, $\mu_{s}-\delta \mu / 2 \leq \mu \leq \mu_{s}+\delta \mu / 2$, and $\mathcal{V}\left(\psi_{r}\right)$ is the specific volume at $\psi_{r}$, defined so that

$$
\int_{0}^{1} \mathcal{V}(\psi) \mathrm{d} \psi=V
$$

is the total volume of the plasma. In practice, however, this approach is bound to fail, and we had to resort to a different method.

To explain the reason of this failure, let us recall that SSFPQL solves equation (1) by expanding the distribution function in Legendre polynomials of the velocity pitch angle,

$$
F_{i}(\psi, v, \mu)=\sum_{n=0}^{N} F_{i}^{(n)}(\psi, v) P_{n}(\mu)
$$

To be implemented in the code, the r.h.s. of eqn (2) must be similarly expanded

$$
\mathcal{S}_{i}^{\mathrm{nbi}}(\psi ; v, \mu)=\sum_{n}\left(\sum_{k=1}^{N_{s}} \frac{\delta\left(v-v_{i, k}\right)}{v_{i, k}^{2}} \frac{\sigma_{i, k}^{(n)}(\psi)}{2 \pi} P_{n}(\mu)-K_{i}^{\mathrm{nbi}} F_{M}(v) \delta_{n, 0}\right)
$$

where in the loss term $F_{M}(v)=e^{-v^{2}} / \pi^{3 / 2}$ is the Maxwellian distribution, and

$$
\sigma_{i, k}^{(m)}(\psi)=\left(m+\frac{1}{2}\right) \int_{-1}^{+1} \mathcal{S}_{i, k}(\psi, \mu) P_{m}(\mu) d \mu
$$


The quantities $\mathcal{S}_{i, k}$ obtained from Monte Carlo simulations, however, are far too noisy to allow the numerical evaluation of the integrals on the r.h.s. of eq. (7). Instead, the coefficients $\sigma_{i, k}^{(l)}(\psi)$ of the expansion of the NBI sources in Legendre polynomials are obtained on each magnetic surface by performing a standard minimum square error fitting of the Monte Carlo data assuming a Gaussian distribution in angle.

The advantage of evaluating the quantities $\mathcal{S}_{i, k}$ from the output of a NBI code not based on the Monte Carlo approach is evident. SINBAD [32] is a fast and accurate NBI code based on a "narrow-beam" model, which takes into account the geometry of the injectors, and the divergence and/or focusing of the beams. The NBI deposition profiles are evaluated as function of the velocity pitch angle and expanded in Legendre polynomials as required by SSFPQL. Optionally, SINBAD averages the initial deposition profiles along the first guiding center orbit of the newborn fast ions: this allows to take into account finite orbits effects and to estimate the prompt losses. The interface between SINBAD and SSFPQL has been described in [36], where the predictions of FAFNER and SINBAD are also compared. They are generally found in good agreement, with some discrepancies near the magnetic axis which, however, do not significantly influence the global power balance.

The input of particle and power density due to the ionization of the beams are

$$
\left(\frac{d n_{i}(\psi)}{d t}\right)_{\mathrm{nbi}}=\sum_{k=1}^{N_{s}} \sigma_{i, k}^{(0)}(\psi)
$$

and

$$
W_{i}^{\mathrm{nbi}}(\psi)=\sum_{k=1}^{N_{s}} E_{i, k} \sigma_{i, k}^{(0)}(\psi)
$$

respectively. To maintain a constant density in the presence of NBI, therefore, in the loss term of eq. (6) we must choose

$$
K_{i}^{\mathrm{nbi}}=\sum_{k} \sigma_{i, k}^{(0)}(\psi)
$$

The corresponding power losses

$$
W_{i}^{\text {loss }}=-\frac{3}{2} \sum_{k} \sigma_{i, k}^{(0)}(\psi) T_{i}
$$

are always very small compared to the power injected by the neutral beams.

With NBI alone the system of equations for the coefficients $F^{(n)}$ in eq. (5) is diagonal

$$
\begin{aligned}
\frac{1}{v^{2}} \frac{d}{d v} & {\left.\left[v^{2}\left(\frac{\Psi_{c}(v)}{2 v} \frac{d F_{i}^{(n)}}{d v}+\Psi_{\tau}(v) F_{i}^{(n)}\right)\right]-n(n+1) \frac{\Theta_{c}(v)}{2 v^{3}} F_{i}^{(n)}\right\}=} \\
= & \mathcal{S}_{\Delta T}^{i}(\psi, v) \delta_{n, 0}+\sum_{k=1}^{N_{s}}\left(\frac{\delta\left(v-v_{i, k}\right)}{2 \pi v_{i, k}^{2}} \sigma_{i, k}^{(n)}(\psi)-\frac{e^{-v^{2}}}{\pi^{3 / 2}} \sigma_{i, k}^{(0)}(\psi) \delta_{n, 0}\right)
\end{aligned}
$$

(the notations for the collisional operator are those of [20]). It is easily seen, moreover, that if the series representing the NBI source is uniformly convergent, then the series 
representing the distribution function also converges uniformly, and actually appreciably faster than the former. When NBI and IC heating are simultaneously present, on the other hand, to obtain a convergent solution the summation (5) for $F_{i}(v, \mu)$ must include a large number of terms. This, in turn, requires the evaluation of integrals involving products of Legendre polynomials of very high order. To avoid the accumulation of rounding errors in the iterative evaluation of these integrals (cfr. the appendix to [20]), tables of these integrals for Legendre polynomials up to order 60 evaluated with Mathematica [37] have been made available. Although reading the integrals from these tables is appreciably slower than evaluating them in Fortran, it has the advantage that the values obtained with Mathematica are 'exact', since they are evaluated with integer arithmetic, except for a final division.

A limitation of the SSFPQL model in the presence of high-power NBI must be mentioned. The linearized collision term implemented in the code adequately describes collisional power exchanges between different kinds of particles, but not collisional exchanges of momentum. The macroscopic velocities caused by NBI do not exceed a few percent of the thermal speed, and are generally small compared to the typical rotation velocities due to the development of a radial electrostatic ambipolar potential. Simulating this rotation is obviously outside the scope of the surface-averaged kinetic equation (1). Nevertheless, it would be more satisfactory to be able to evaluate accurately also collisional exchanges of momentum. For this purpose one should include in the model appropriate additional terms in the Legendre expansion of the TrubnikovRosenbluth potentials [38]. These terms, however, make the collision operator non linear, although weakly, and would thus require a non-negligible rewriting of the code.

\section{A synthetic neutron rate diagnostics}

When the fast ions are reactants of nuclear fusion reactions with neutron yield (in particular the reaction $\left.\mathrm{D}+\mathrm{D} \rightarrow \mathrm{He}^{3}(0.82 \mathrm{MeV})+\mathrm{n}(2.45 \mathrm{MeV})\right)$, the enhancement of the neutron rate can be used to infer the effectiveness of energetic ion creation by NBI and/or IC heating. Since SSFPQL represents the distribution functions as an expansion in Legendre polynomials, it is natural to calculate the neutron rate with the algorithm for the reactivity $\langle\sigma v\rangle$ suggested by Cordey and co-workers [33]. The fusion reaction rate between two species of density $n_{a}$ and $n_{b}$ is defined as

$$
\mathcal{R}=n_{a} n_{b}<\sigma v>=n_{a} n_{b} \int \mathrm{d} \vec{v} \int \mathrm{d} \vec{v}^{\prime} F_{a}(\vec{v}) F_{b}\left(\vec{v}^{\prime}\right) \sigma\left(\left|\vec{v}-\vec{v}^{\prime}\right|\right)\left|\vec{v}-\vec{v}^{\prime}\right|
$$

(the distribution functions $F_{a}$ and $F_{b}$ are supposed normalized to unity). Inserting the Legendre polynomial expansion (5) for $F_{a}$ and $F_{b}$, reduces the six-dimensional integral (13) to a triple sum

$$
<\sigma v>=\sum_{j, j^{\prime}=1}^{N_{v}} \sum_{n=0}^{N_{L}} F_{a}^{(n)}\left(v_{j}\right) F_{b}^{(n)}\left(v_{j^{\prime}}\right) I_{j j^{\prime}}^{(n)}
$$


with

$$
I_{j j^{\prime}}^{(n)}=c_{j} c_{j^{\prime}} \frac{8 \pi^{2}}{2 n+1} v_{j}^{2} v_{j^{\prime}}^{2} \sum_{k} P_{n}\left(\mu_{k}\right) c_{k} \sigma\left(u_{j j^{\prime} k}\right) u_{j j^{\prime} k}
$$

Here $u_{j j^{\prime} k}=\sqrt{v_{j}^{2}+v_{j^{\prime}}^{2}-2 v_{j} v_{j^{\prime}} \mu_{k}}, \mu_{k}$ is the cosine of the angle between $\vec{v}_{j}$ and $\vec{v}_{j^{\prime}}$, and the coefficients $c_{k}$ are the weights of the quadrature formula used for integration. The quantities $I_{j j^{\prime}}^{(n)}$ need to be calculated only once on a velocity mesh large enough to cover the largest velocity mesh to be used in SSFPQL. For the cross section $\sigma$ we have implemented the Bosch-Hale parametric formula (eqn (8) of [39]), with the values of the parameters given in Table IV of this reference.

\section{Fast ion losses during slowing down}

As mentioned in [20], an important limitation of the kinetic equations solved by SSFPQL was the absence of a term describing losses of fast ions during slowing down. The inclusion of fast ion (FI) losses is particularly important when simulating experiments in relatively small tokamaks, in which very energetic ions are unlikely to be confined for times long enough to reach full thermalization (these losses should not be confused with prompt losses of ions born or pushed by quasilinear interactions on unconfined orbits). Without a FI losses term, the synergies between IC and NBI appear to be largely overestimated.

For a really satisfactory description of FI losses one should add to the kinetic equation a radial diffusion term with energy dependent diffusion coefficient [40]. This, however, would substantially increase the difficulty of the numerical implementation. The mechanisms responsible for this diffusion, moreover, can range from neoclassical and rf-induced diffusion, to resonant interactions with low frequency instabilities and drift turbulence, or with microinstabilities excited by the free energy present in the quasilinear distributions themselves. Each of these mechanisms can result in a radial diffusion with a different velocity-space dependency. These effects are best studied and modeled with dedicated Monte Carlo codes. Our goal here can only be to estimate the importance of such losses. In this we are helped by the fact that when solving directly for the steady-state, as with SSFPQL, a loss term with appropriately chosen coefficients can in principle simulate the effects of radial diffusion with sufficient accuracy.

To model losses of energetic ions during thermalization we have, therefore, implemented an heuristic loss term fulfilling two constraints, namely that the total number of particles must be conserved to allow for a steady-state, and that the Maxwellian $F_{M}(v)$ must be a solution of (1) also in the presence of FI losses:

$$
\begin{aligned}
\mathcal{L}_{i}^{\mathrm{FIL}}(\psi, v, \mu) & =\frac{1}{v^{2}}\left\{R_{i}(\psi) F_{M}(v)\right. \\
& \left.-B_{i}(\psi) T(\mu, \psi) U(v, \psi)\left[F_{i}(v, \mu, \psi)-F_{M}(v)\right]\right\}
\end{aligned}
$$


whose Legendre representation has coefficients

$$
\begin{aligned}
\mathcal{L}_{i}^{\mathrm{FIL}(n)}(\psi, v) & =\frac{1}{v^{2}}\left\{R_{i}(\psi) F_{M}(v) \delta_{n, 0}\right. \\
- & \left.B_{i}(\psi) U(v) \sum_{n^{\prime}} T_{n, n^{\prime}}(\psi)\left[F_{n^{\prime}}(v)-F_{M}(v) \delta_{n^{\prime}, 0}\right]\right\}
\end{aligned}
$$

where

$$
T_{n, n^{\prime}}(\psi)=\left(n+\frac{1}{2}\right) \int_{1}^{1} T(\mu, \psi) P_{n}(\mu) P_{n^{\prime}}(\mu) \mathrm{d} \mu
$$

The existence of a steady-state solution imposes a 'refuelling rate'

$$
R_{i}(\psi)=\pi B_{i}(\psi) \sum_{n^{\prime}} T_{0, n^{\prime}}(\psi) \int_{0}^{\infty} U(v)\left[F_{n^{\prime}}(v)-F_{M}(v) \delta_{n^{\prime}, 0}\right] d v
$$

(in the Maxwellian limit $R_{i}(\psi)=0$ ). The energy losses associated with $\mathcal{L}_{i}^{\text {FIL }}$ are

$$
\begin{aligned}
& \frac{1}{\nu_{i} n_{i} T_{i}}\left(\frac{d n_{i} E_{i}}{d t}\right)_{\text {loss }}=\frac{R_{i}(\psi)}{2} \\
& \quad-4 \pi B_{i}(\psi) \sum_{n^{\prime}} T_{0, n^{\prime}}(\psi) \int_{0}^{\infty} U(v)\left[F_{n^{\prime}}(v)-F_{M}(v) \delta_{n^{\prime}, 0}\right] v^{2} \mathrm{~d} v
\end{aligned}
$$

with $\nu_{i}$ the collision frequency of species $i$. The energy gain due to the refuelling term is always much smaller than the energy lost by suprathermal ions. The loss term $\mathcal{L}=F / \tau(\psi)$, frequently used to model losses in presence of $\operatorname{ICRF}[13,34,35,19]$, can be obtained as a special case of (16).

In the absence of better information, we have kept the FI loss term as simple as possible by making the following assumptions:

- The dependence of FI losses from the velocity pitch angle has been assumed to be of the form

$$
T_{i}(\mu, \psi)=\left(1-\mu^{2}\right)^{p} \quad 0 \leq p \leq 3
$$

With $p>0$ this allows to localize the losses to some extent in the trapped orbits domain. The advantage of this form is that the coefficients (18) can be evaluated in closed form in terms of integrals of the same kind as those used to build the QLO [20].

- For the velocity dependence of FI losses we have assumed a smoothed step function

$$
U_{i}(v)=\left\{\begin{array}{ll}
0 & \text { if } v \leq v_{\mathrm{f}} \\
\frac{y(u)}{1+y(u)} & \text { if } v>v_{\mathrm{f}}
\end{array} \quad y(u)=\left(\frac{v-v_{\mathrm{cr}}}{a_{\mathrm{f}}}\right)^{k}\right.
$$

with $k \geq 2$ an integer, $v_{\mathrm{f}} \gtrsim v_{t h i}$ and $a_{\mathrm{f}} \gtrsim v_{t h i}$ such that relatively energetic ions are preferentially lost before being thermalized.

- The radial dependence has been taken to be

$$
B_{i}(\psi)=K_{\mathrm{FIL}}\left[\xi+(1-\xi) \psi^{q}\right]
$$




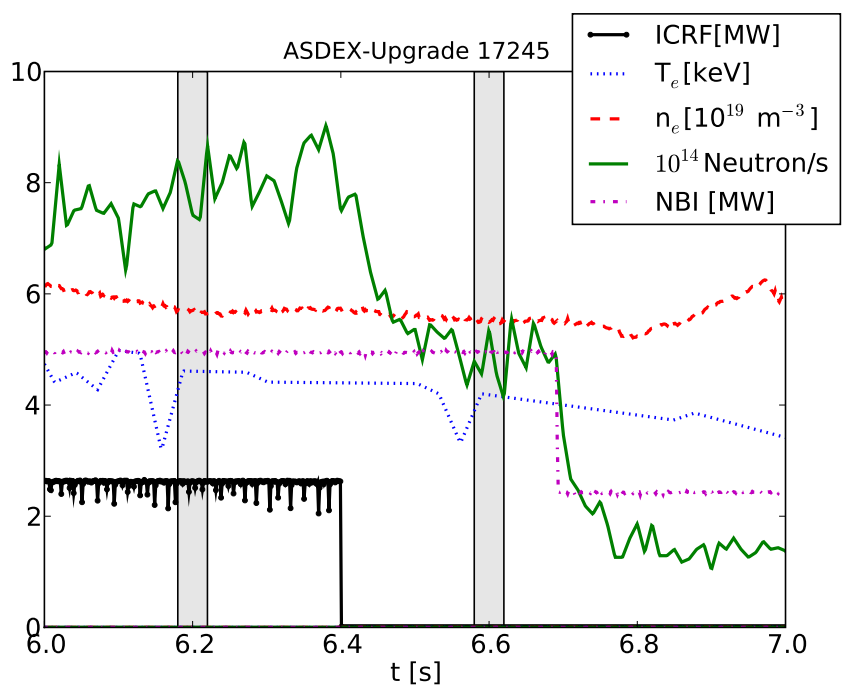

Figure 1. Time traces of the discharge 17245 of ASDEX Upgrade.

with $K_{\mathrm{FIL}}$ and $\xi \leq 1$ constants, $q>0$ but not necessarily an integer. A dependence of the constant $K_{\mathrm{FIL}}$ on the mass to charge ratio $A_{i} / Z_{i}$ of the ions is plausible, but obviously not proved.

\section{Examples.}

We present now a few simulations by the TORIC-SSFPQL package of an ASDEX Upgrade ([41]) discharge (shot 17245) in which both NBI and IC heating where applied. These simulations are primarily aimed at testing the code and understanding its predictions; nevertheless, they also allow to draw some interesting conclusions.

The discharge 17245 of ASDEX Upgrade was characterized by a $3 \%$ Hydrogen minority concentration in a Deuterium plasma. Non negligible concentrations of Carbonium $\left(Z_{\text {eff }} \simeq 3[42]\right)$ and Helium where also present, the latter due to previous vessel cleaning with this gas. Two NBI sources injected Deuterium with energies centered at 60 and $93 \mathrm{keV}$, respectively, with contributions at $1 / 2$ and $1 / 3$ of these values, carrying respectively $28 \%$ and $9 \%$ of the total NBI power. Figure 1 summarizes a few of the experimental time traces of this discharge, namely the central electron density and temperature, the neutron rate, and the wave-form of the NBI and ICRF power applied. We have selected two time slices, at $t=6.2$ and $t=6.6 \mathrm{sec}$, respectively. At $t=6.2 \mathrm{sec}$ NBI $(\sim 5 \mathrm{MW})$ and ICRF $(\sim 2.5 \mathrm{MW})$ where simultaneously applied; later only NBI was present, namely again $\sim 5 \mathrm{MW}$. According to the relatively well documented diagnostics, the plasma parameters did not appreciably change between these two times. We assume this to be true also of the plasma composition. The radial profile of the NBI power deposition calculated by FAFNER is shown in fig. 2 for the two time slices. The sources calculated by SINBAD are nearly identical. 


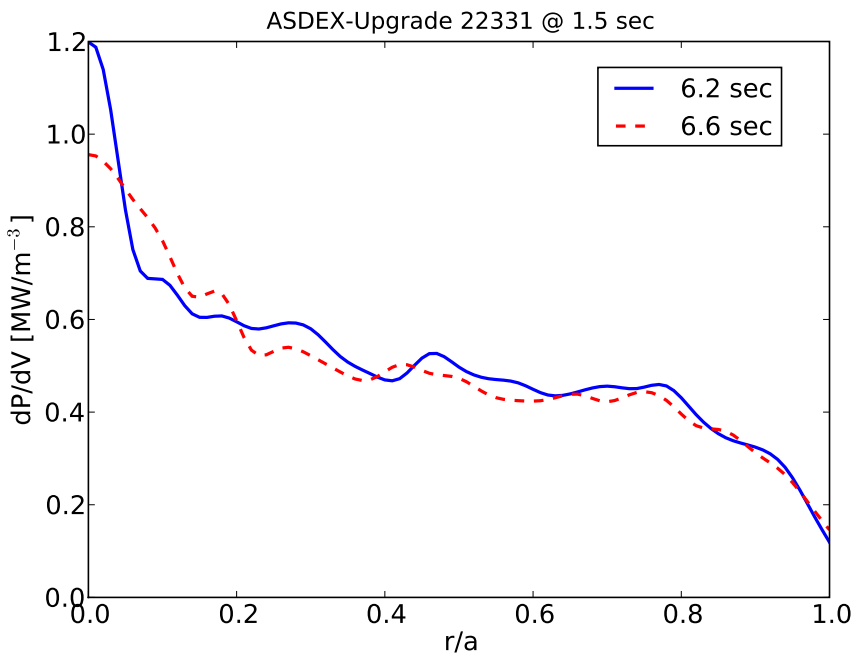

Figure 2. Radial NBI power deposition profiles at $t=6.2 \mathrm{sec}$ (blue) and $t=6.6 \mathrm{sec}$ (red) calculated by FAFNER code.

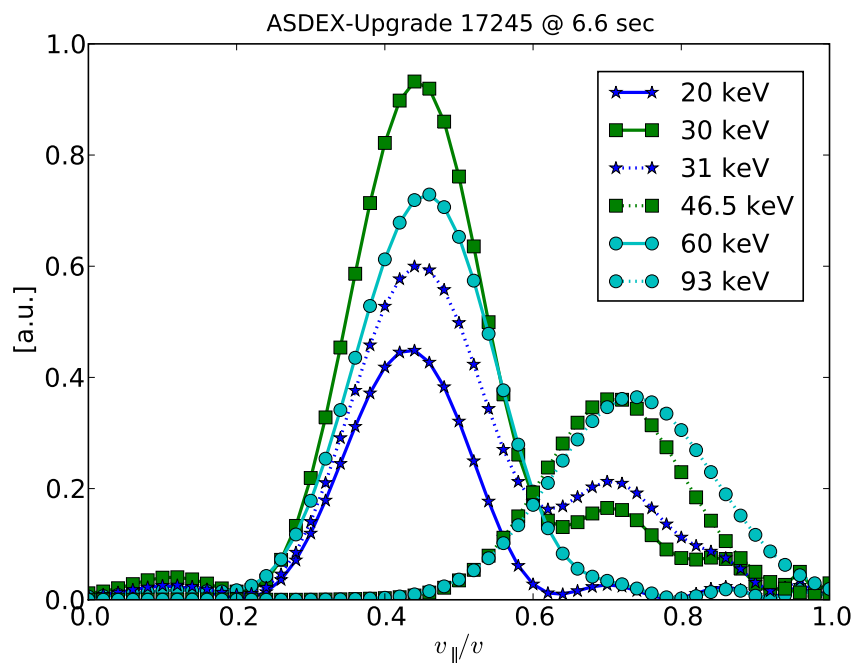

Figure 3. Legendre reconstruction of the pitch-angle distribution of the NBI sources at $r / a=0.2$ (AUG discharge $17245, t=6.6$ ). Only the range $v_{\|}>0$ is plotted, since the beams where oriented for coinjection.

\subsection{Neutral Beam Injection alone}

Let us begin by considering the latter time slice with $4.8 \mathrm{MW}$ of NBI applied alone (prompt losses were estimated to be about $0.2 \mathrm{MW}$ ). Figure 3 shows the angular dependence of the NBI sources at the plasma center, reconstructed from their Legendre representation. The Deuterium distribution function predicted by SSFPQL is shown in fig. 4. Distortion from Maxwellian is largest at a velocity pitch-angle $\mu \simeq+0.5$, in agreement with the somewhat oblique coinjection. Beyond the plateau caused by the sources, the Maxwellian slope is rapidly reached again, as one would expect. If a 


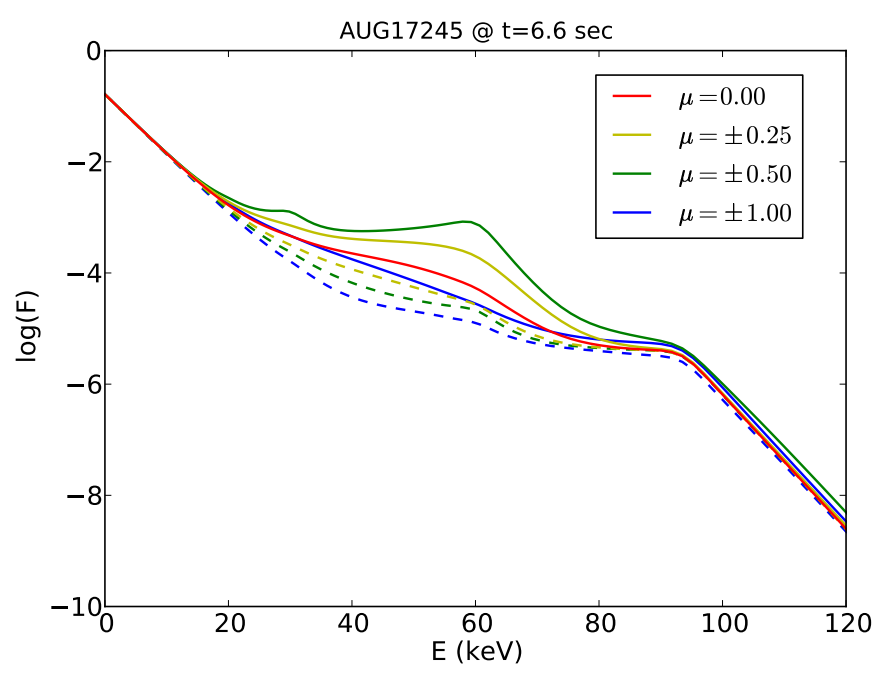

Figure 4. Semilogarithmic plot of the Deuterium distribution function on axis for a few values of the velocity pitch angle (full lines: $v_{\|} / v>0$, dashed lines $v_{\|} / v<0$; AUG discharge 17245, $t=6.6 \mathrm{sec}$, 4.8 MW NBI, without Fast Ion Losses).

pure D-H plasma were assumed, the predicted integrated neutron rate without FI losses was $9.2 \times 10^{14}$ neutron/sec, a factor two larger than the experimental value, namely $5 \times 10^{14}$ neutron/sec (the rate of the unperturbed Maxwellian plasma is estimated to be $\sim 1.25 \times 10^{12} / \mathrm{sec}$ ). With sufficient Carbon to make $Z_{\text {eff }}=3$, on the other hand, the predicted neutron rate was only $20 \%$ higher than the measured value. The discrepancy decreases further if Helium is included, the experimental value being obtained assuming a concentration $\left(100 n_{i} / n_{e}\right)$ of $5 \%$, as shown in fig. 5 . Since with NBI alone the suprathermal ion energies do not exceed 100 to $120 \mathrm{keV}$ (fig. 4), and there was no experimental indication of intense losses of fast ions, we will assume this to be the plasma composition.

\subsection{Simultaneous Neutral Beam Injection and Ion Cyclotron heating}

At time $6.2 \mathrm{sec}$ in the same discharge $4.8 \mathrm{MW}$ of NBI and nominally $2.5 \mathrm{MW}$ of ICRH were simultaneously applied, resulting in a neutron production of $\sim 8 \times 10^{14} / \mathrm{sec}$. The magnetic field on axis $\left(B_{0}=2.5\right.$ Tesla) and the frequency $(f=36.5 \mathrm{MHz})$ were such that the fundamental IC resonance of $\mathrm{H}$ and the first IC harmonic of D where located quite close to the magnetic axis. As shown in fig. 6, the neutron production predicted by TORIC-SSFPQL when FIL are omitted (1.2 $10^{15}$ per sec) exceeds the experimentally measured value by $50 \%$. The predicted value, if the results are taken face-value, might even be underestimated: although in these simulations we have extended the integration range to cover energies up to $0.75 \mathrm{MeV}$, at powers above $1.5 \mathrm{MW}$ there would be sufficient ions above this energy to give a significant additional contribution to the neutron production. In ASDEX Upgrade, however, Deuterons with energies exceeding about $0.5 \mathrm{MeV}$ have orbits so large that their description by a surface-averaged kinetic 


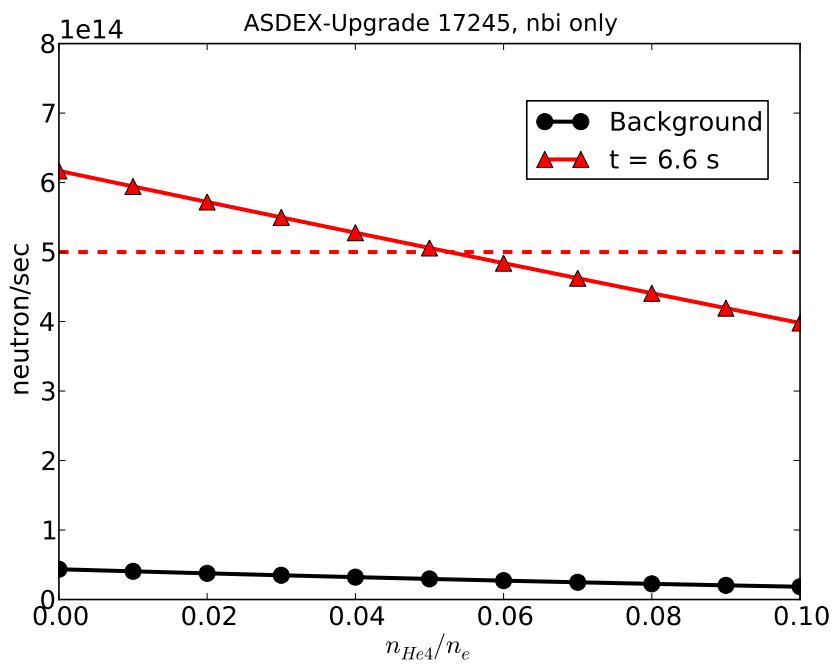

Figure 5. Predicted neutron count versus $\mathrm{He}^{4}$ concentration; AUG discharge 17245, $t=6.6 \mathrm{sec}, 4.8 \mathrm{MW}$ NBI, without Fast Ion Losses).
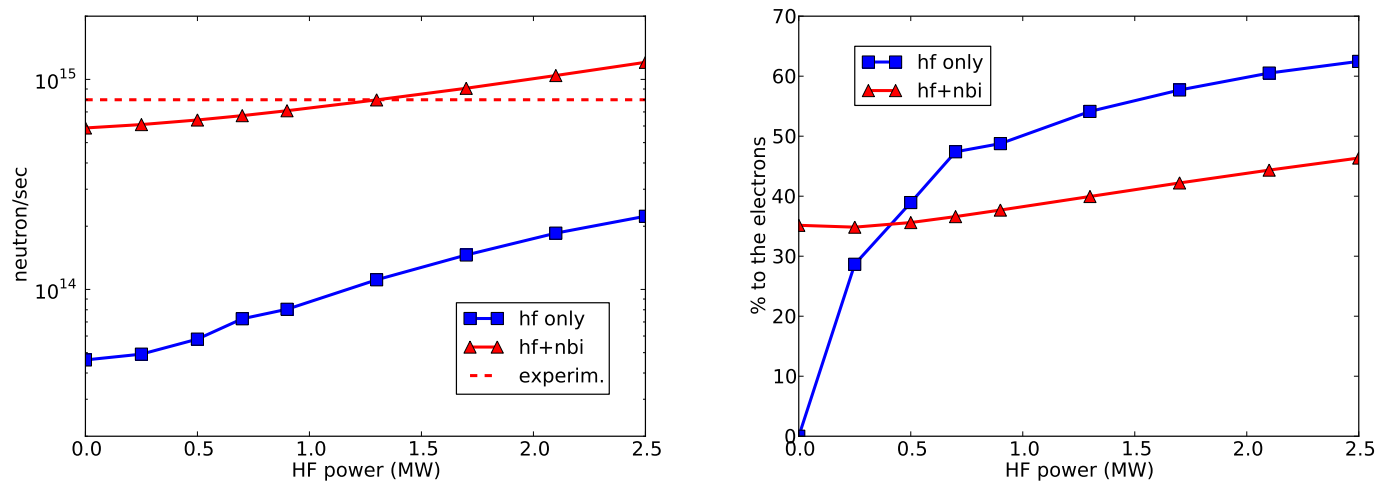

Figure 6. Comparing hf heating alone and hf heating simultaneous with NBI Left: Neutron count; Right: fraction of power collisionally transferred to the electrons.

equation becomes questionable.

Although under these circumstances the omission of FIL is not very realistic, it is nevertheless worth presenting some more details of these simulations. In figs. 7 we compare the power absorption profiles for a Maxwellian plasma, and with the quasilinear distribution functions evaluated by SSFPQL for a total hf power of 2.1 MW, using in both cases the measured density and temperature profiles. In the selfconsistent run the absorption profile of both Deuterium and Hydrogen are appreciably broader than in the Maxwellian case; the domain of absorption by Hydrogen, moreover, is pushed to the outside, since near the center the development of strong suprathermal tails makes harmonic damping more competitive with respect to absorption at the fundamental. The number of suprathermal protons, although sufficient to increase the partial pressure of Hydrogen by more than a factor 2, is nevertheless less than one per cent of the total 

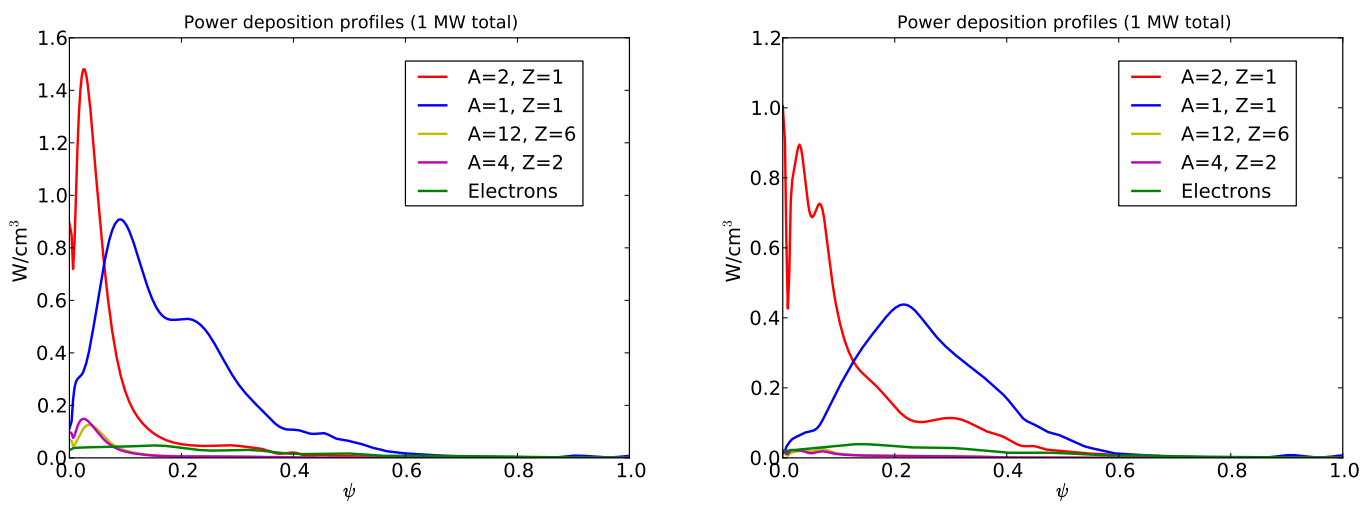

Figure 7. : Power deposition profiles of $2.1 \mathrm{MW}$ of hf power in the presence of 5 MW of NBI. Left: Maxwellian plasma, right: selfconsistent quasiilinear distribution functions.
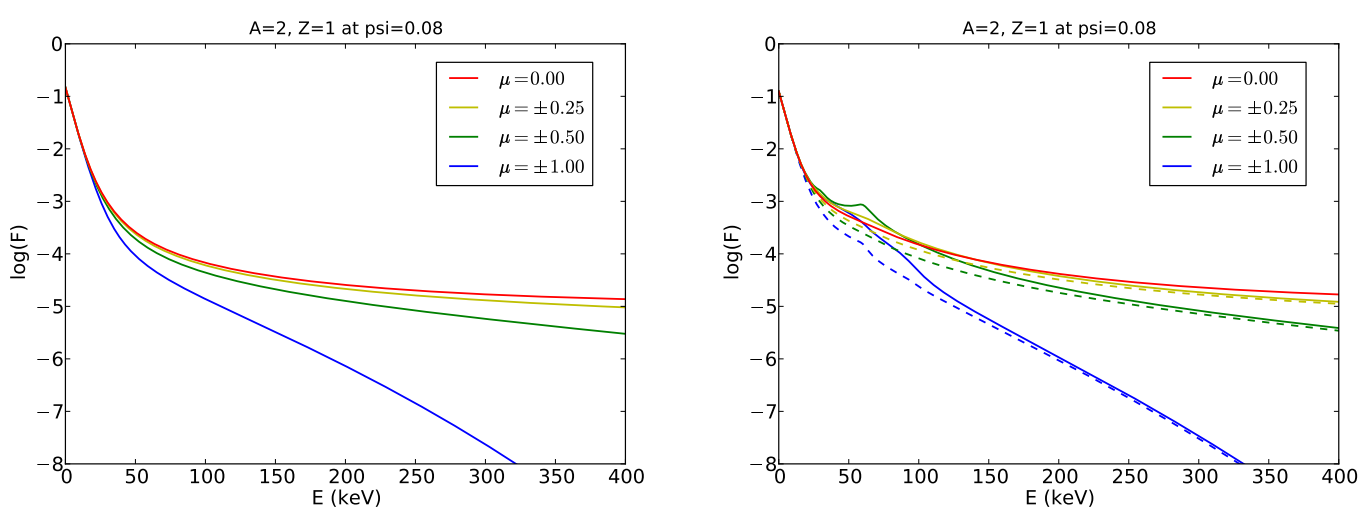

Figure 8. Comparing hf heating alone and hf heating simultaneous with NBI, Deuterium distribution function at different pitch-angles at the point of peak absorption. Left: hf heating only (2.1 MW); Right: hf heating $(2.1 \mathrm{MW})$ and NBI (5 MW).

Hydrogen concentration. Energetic Deuterons are an even smaller fraction (less than one in a thousand) of the Deuterium concentration, but this is more than compensated by the fact that Deuterium is the majority, and by the strong dependence of harmonic IC damping on the ion energy. As a result, the fraction of hf power absorbed by Deuterium increases from $14.4 \%$ in the Maxwellian case to $28.2 \%$ when the quasilinear distributions are selfconsistently taken into account, mainly at the expense of Hydrogen.

The synergies between NBI and harmonic IC heating are best illustrated by comparing the predicted neutron count and the fraction of power collisionally transmitted to the electrons in steady-state versus hf power with and without simultaneous NBI (fig. 6). A quantitative characterization of these synergies is difficult, but the neutron production with the two heatings applied simultaneously is larger than the sum of those by each method separately applied. In the Deuterium distribution 

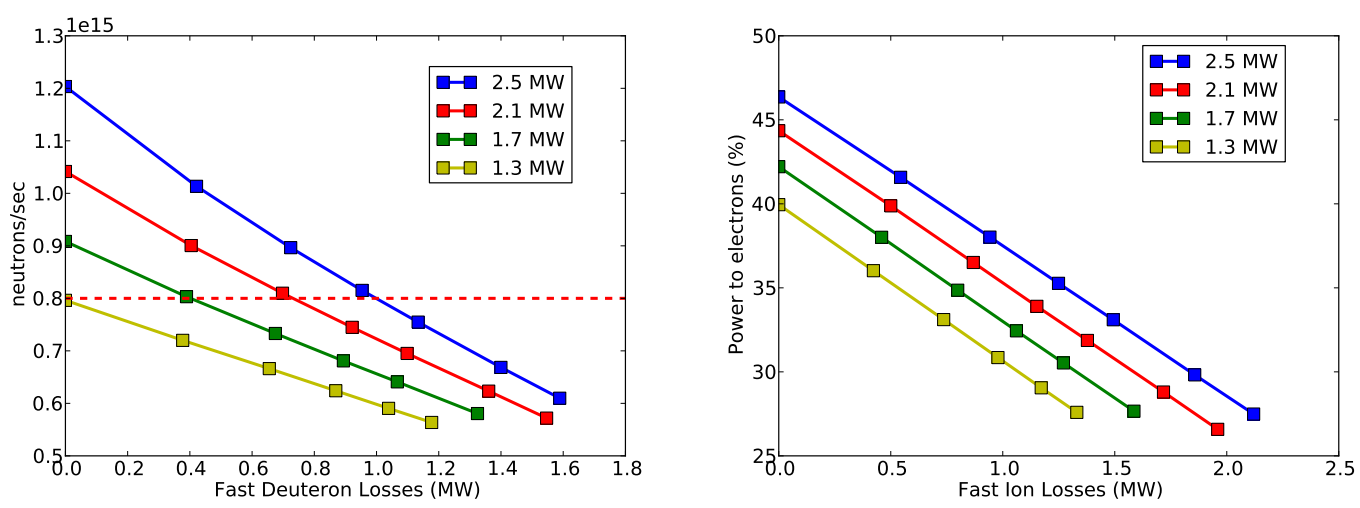

Figure 9. The effect of fast ion losses during thermalization. Left: ; Right: fraction of power collisionally transferred to the electrons. (2.1 MW) and NBI (5 MW).

function the largest increase of high-energy ions is in the range from 100 to $250 \mathrm{keV}$ (fig. 8), just above the energy of the NBI sources. Because of the logarithmic scale, this increase is not immediately apparent from the figure; a detailed comparison, however, shows that in this range the number of energetic ions increases by a factor of 2 to 5 in the presence of NBI. This is also the energy range of largest cross-section for neutron production. In particular, the relatively large increment of $F(E)$ in the parallel direction has a non negligible influence on wave propagation, by increasing the Doppler width of the Deuterium harmonic resonance. Above $\sim 250 \mathrm{keV}$, on the other hand, the effects of NBI are minimal. We note, however, that the synergy would be much stronger in a plasma with less impurities, in particular, without Helium. The simulations also predict a reduction of the collisional power transfer to the electrons in the presence of NBI, due to the decrease of the the effective temperature of the Hydrogen suprathermal population, imputable to the outwards shift of the Hydrogen peak absorption. Such quasilinear interactions between different species depend sensitively on the location of the resonance layers with respect to the magnetic axis.

As already mentioned, fig. 6 also shows that in the case of simultaneous hf and NBI heating losses of fast ions during slowing down are likely to play a non-negligible role in the global balance. Without such losses, the experimental neutron count is reproduced only if less than 1.5 MW of hf power are deposited in the plasma. Although a fraction of the nominal power may be lost at the plasma periphery, this figure is unlikely low. In ASDEX Upgrade, on the other hand, confinement of ions of several hundred keV is unlikely to be long enough to allow a complete thermalization without some losses. Figure 9 shows that assuming 2.1 MW of hf power to be deposited in the plasma, the experimental neutron count is reproduced if about $0.7 \mathrm{MW}$ are lost by fast Deuterons during thermalization. This figure appears high if compared to the hf power absorbed by the Deuterium (0.56 MW), but is quite reasonable if one takes into account that Deuterium also receives almost $5 \mathrm{MW}$ of NBI power. Losses in the other ion species are much smaller, in the case of Hydrogen (about $170 \mathrm{~kW}$ ) simply because of the very low 

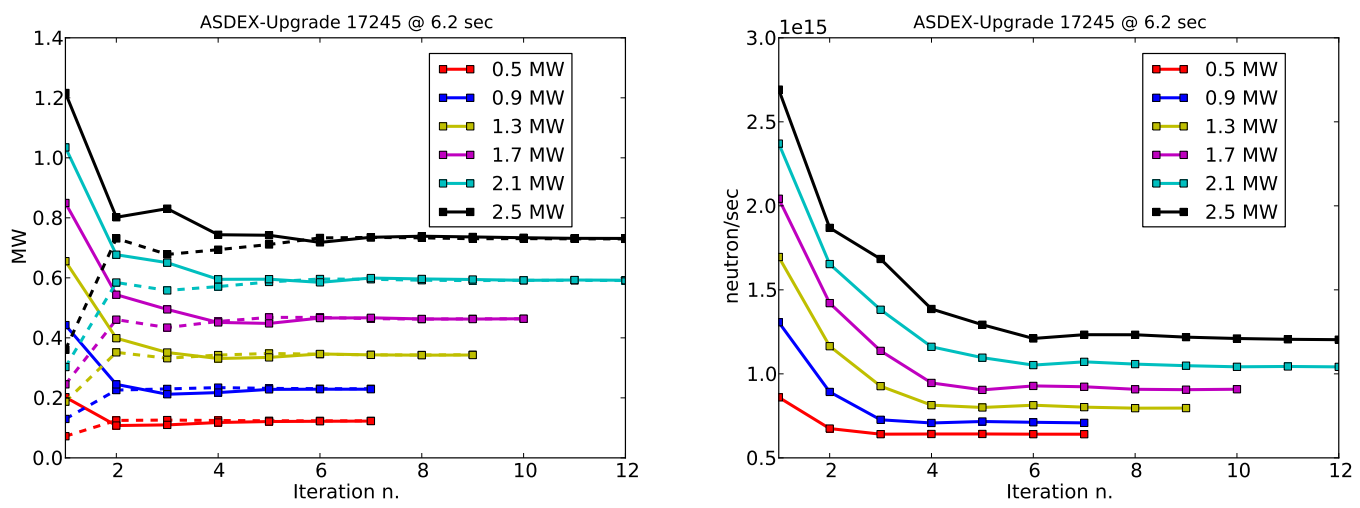

Figure 10. Iteration TORIC-SSFPQL: High Frequency power absorbed by Deuterium (left; full lines: SSFPQL predictions, dashed lines: TORIC predictions) and total neutron count (right) in the presence of $5 \mathrm{MW}$ of NBI, at different levels of the total hf power.

proton concentration.

\subsection{Iterating between TORIC and SSFPQL.}

To complete the discussion, it might be of some interest to present some details of the iteration between the two codes. Maxwell's equations and the quasilinear kinetic equations predict the same power absorption when the distribution functions are the same. The expressions to be coded, however, although equivalent in principle, are quite different: in TORIC absorption is proportional to the antihermitian part of the coefficients of the wave equations, while in SSFPQL it is evaluated as a moment of the distribution function weighted with the quasilinear diffusion coefficient. Iterating successfully between the two codes, therefore, is not an entirely trivial task. At high power, in this first iteration the total absorption estimated by the two codes may differ by 10 to $25 \%$ and more. These global values, moreover, often result from internal compensations: the discrepancy for individual species can exceed a factor two. In the applications presented here the iteration between TORIC and SSFPQL has been stopped when the integrated power absorbed estimated by the two codes differ by less than $1 \%$ separately for each ion species. To obtain convergence in a reasonable number of iterations, it was necessary to improve substantially the accuracy of the evaluation of the coefficients of the wave equations to be transferred to TORIC [20].

We illustrate this convergence for one of the examples presented in the this section, namely the case at $t=2.1 \mathrm{sec}$, assuming $2.1 \mathrm{MW}$ of hf power to be deposited in the plasma together with NBI at $5 \mathrm{MW}$; no losses of fast ions were taken into account. Figure 10 shows the evolution with the iteration number of the high frequency power absorbed by Deuterium and of the total neutron count, at different levels of the total hf power. At the first iteration the hf power deposited in the Deuterium according to SSFPQL is almost twice that predicted for a Maxwellian plasma; this is because 

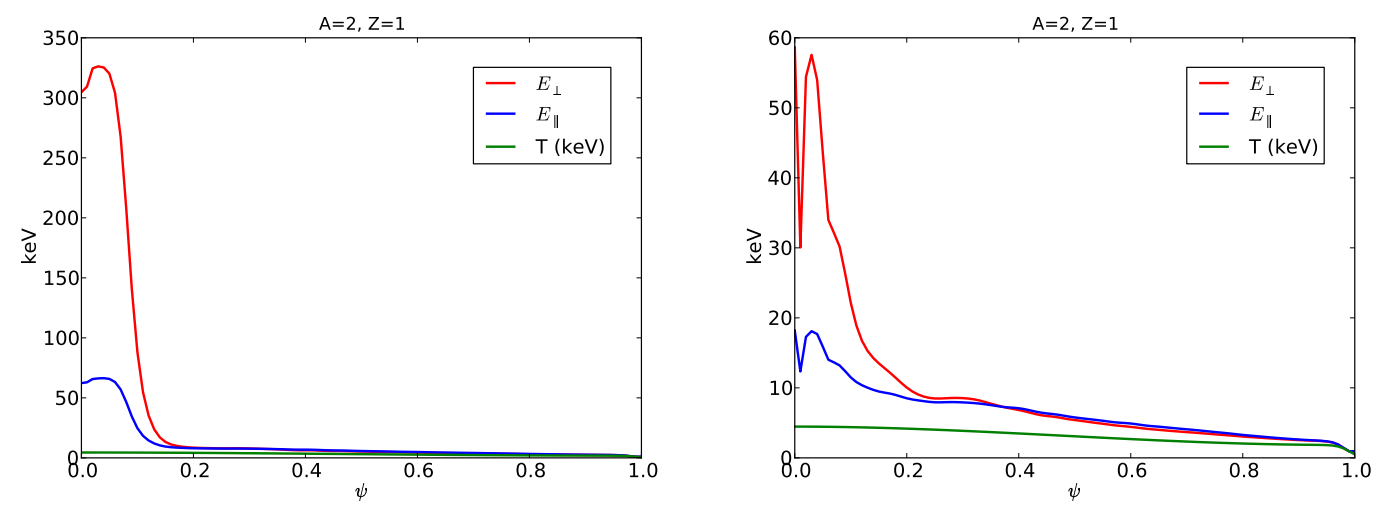

Figure 11. Iteration TORIC-SSFPQL: Perpendicular and parallel averaged energy in Deuterium ions. Left: First iteration, right: convergent iteration.

SSFPQL evaluates the power deposition with strongly suprathermal quasilinear tails obtained using the fields of the first run of TORIC, in which, by contrast, Maxwellian ions were assumed. In the next iteration TORIC takes into account the presence of these tails: the global absorption by Deuterium is found to be larger than in the Maxwellian case, but the Doppler broadening of the absorption peak causes the power per unit volume to decrease. With the broadened power deposition profiles the tails predicted by SSFPQL are now somewhat weaker, and this guarantees that the iteration converges in spite of the self-boosting nature of harmonic IC heating. The converged hf power deposited in the Deuterium is about half-way between the initial predictions of TORIC and SSFPQL. Accordingly, the converged total neutron count is only just half of the initial prediction. Even more dramatic is the evolution of the predicted average energy of the distribution functions with iteration number (figs. 12 and 11). In the case of Deuterium the first run of SSFPQL greatly over-estimates the energy content of the suprathermal population. The local increase in the plasma pressure and anisotropy predicted by this first run would hardly be compatible with the maintenance of the Grad-Shafranov MHD equilibrium. The convergent values are much more moderate, although far from negligible. The importance of a convergent iteration for quantitative predictions is evident from these results.

\section{Discussion and conclusions}

Although some limitations, due in particular to surface-averaging, have to be taken into account, coupling the SSFPQL solver of the quasilinear kinetic equations with the TORIC full-wave solver of Maxwell equations in toroidal geometry is a flexible and efficient tool for selfconsistent simulations of plasma heating in tokamaks in the IC range of frequencies. The implementation of a source describing NBI in SSFPQL and improvements of numerical accuracy of the code have further extended the possible applications of this package.

To test the performances of the two codes for the investigation of NBI and 

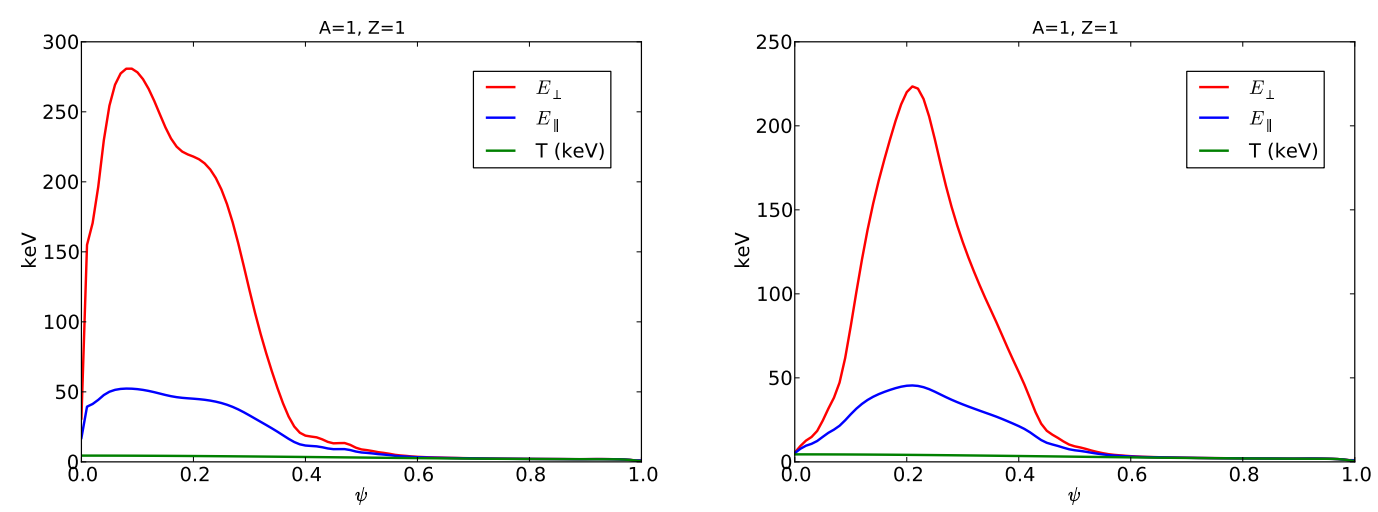

Figure 12. Iteration TORIC-SSFPQL: Perpendicular and parallel averaged energy in Hydrogen ions. Left: First iteration, right: convergent iteration.

IC simultaneous heating we have compared their predictions with experimental results obtained on the ASDEX Upgrade tokamak. In order to reproduce the experimental measurements in discharges where the two heating methods where applied simultaneously, an heuristic loss term describing losses of energetic ions during slowing down had to be added to SSFPQL. To fit simulations and experiments we have heavily used, in particular, the comparison between neutron rate by $\mathrm{D}-\mathrm{D}$ collisions measured and estimated with the synthetic diagnostics included in SSFPQL. This diagnostics is very sensitive to suprathermal Deuterons.

In addition to give useful information on the losses of fast ions during slowing down, on which not much is known otherwise, the simulations have shown that the knowledge of the plasma composition is a critical issue in evaluating the synergies between IC and NBI heating. In the discharge we have analyzed, dilution by ${ }^{4} \mathrm{He}$ appears to have played an important role in reducing the neutron production, although not entirely suppressing the need of taking into account losses of fast ions during thermalization. We have also noted that accurate measurements of the power collisionally transferred to the electrons could be very useful to improve the understanding of these losses and to check the consistency of the simulations. Perhaps the most important conclusion from this analysis, however, is the fact that the experimentally study of synergies between IC and NBI heating would require a plasma as free as possible from low-Z impurities.

The example analyzed shows the importance of a fully converged iteration between the full-wave and the Fokker-Planck solver, with sufficient resolution both in real and velocity space, particularly in the presence of simultaneous ICRF and NBI heating. The codes TORIC and SSFPQL, with their moderate requirement of computing resources, offer an efficient tool for this purpose.

\section{Acknowledgments}

The TORIC code has benefited from important contributions of P. T. Bonoli, E. D'Azevedo, D. McCune, F. Meo, and J. C. Wright and from fruitfull discussions 
with A. Cardinali. We are particularly indebted to Y. Feng, who kindly provided us with the source of his SINBAD code, and we thank G. Tardini for providing benchmark cases for testing the version of SINBAD adapted to the SSFPQL code. We are also grateful to E. Poli for his careful reading of the manuscript and his useful comments. 
[1] STIX, T. H., Plasma Physics 14 (1972) 367.

[2] STIX, T., Nuclear Fusion (1975) 737.

[3] KIMURA, H., FUJII, T., SAIGUSA, M., et al., Plasma Physics and Controlled Fusion 35 (1993) 845.

[4] Mantsinen, M. J., MAYORAL, M.-L., KIPTILY, V. G., et al., Phys. Rev. Lett. 88 (2002) 105002.

[5] HeIDBRinK, W. W., LUO, Y., BURRELL, K. H., et al., Plasma Physics and Controlled Fusion 49 (2007) 1457.

[6] Hellesen, C., JOHnSOn, M. G., SUnDEN, E. A., et al., Nuclear Fusion 50 (2010) 022001 (5pp).

[7] LIU, D., HEIDBRINK, W. W., PODESTA, M., et al., Plasma Physics and Controlled Fusion 52 (2010) 025006 (17pp).

[8] OKANO, K., INOUE, N., and UCHIDA, T., Nuclear Fusion 23 (1983) 235.

[9] HEIDBRINK, W. and SADLER, G., Nuclear Fusion 34 (1994) 535.

[10] KOCH, R., WASSEnOVE, G. V., EESTER, D. V., et al., Plasma Physics and Controlled Fusion 37 (1995) A291.

[11] PEKKARI, L.-O., ANDERSON, D., HAMnEN, H., and LISAK, M., Nulcear Fusion 25 (1983) 781.

[12] PEKKARI, L.-O., Nulcear Fusion 25 (1985) 165.

[13] ANDERSON, D., CORE, W., L.G.-ERIKSSON, et al., Physica Scripta 37 (1986).

[14] YAMAGIWA, M., TAKIZUKA, T., and KISHIMOTO, Y., Nuclear Fusion 27 (1987) 1773.

[15] YAmagiWA, M., TAKIZUKA, T., KIMURA, H., and AZUMI, M., Plasma Physics and Controlled Fusion 30 (1988) 943.

[16] HAMAMATSU, K., AZUMI, M., KISHIMOTO, Y., et al., Nuclear Fusion 29 (1989) 147.

[17] JAEgER, E. F., BERRY, L. A., AHERN, S. D., et al., Physics of Plasmas 13 (2006) 056101.

[18] MURAKAMI, S., FUKUYAMA, A., AKUTSU, T., et al., Nuclear Fusion 46 (2006) S425.

[19] LERCHE, E., EESTER, D. V., KRASILNIKOV, A., et al., Plasma Physics and Controlled Fusion 51 (2009) 044006 (22pp).

[20] BRAMBILla, M. and BILATO, R., Nuclear Fusion 49 (2009) 085004.

[21] CHOI, M., GREen, D., HEIDBRINK, W. W., et al., Physics of Plasmas 17 (2010) 056102.

[22] JUCKER, M., GRAVES, J., COOPER, W., et al., Computer Physics Communications 182 (2011) 912 .

[23] BRAmBILla, M., Plasma Physics and Controlled Fusion 41 (1999) 1.

[24] ZONCA, F. and CHEN, L., Physics of Plasmas 7 (2000) 4600.

[25] ERIKSSON, L.-G. and SCHNEIDER, M., Physics of Plasmas 12 (2005) 072524.

[26] Belmondo, V., BILATO, R., BRAmbilla, M., and MAJ, O., Journal of Physics: Conference Series 260 (2010) 012001.

[27] Wright, J. C., BOnoli, P. T., D’AZEvedo, E., and BRAmbilla, M., Computer Physics Communications 164 (2004) 330.

[28] BRAmBilla, M., Plasma Physics and Controlled Fusion 44 (2002) 2423.

[29] WRIGHT, J., BERRY, L., BONOLI, P., et al., Nuclear Fusion 45 (2005) 1411.

[30] BRAMBILlA, M., Nuclear Fusion 34 (1994) 1121.

[31] LISTER, G. G., A fully 3d neutral beam injection code using monte carlo methods, Technical Report 4/222, Max-Planck Institut für Plasmaphysik, Garching, 1985.

[32] FEnG, Y., WOLle, B., and HÜBNER, K., Computer Physics Communications 88 (1995) 161.

[33] CORDEY, J. G., MARX, K. D., MCCOY, M. G., and ET AL., J. Comp. Phys. 28 (1978) 115.

[34] ITOH, K., ITOH, S.-I., and FUKUYAMA, A., Nuclear Fusion 28 (1988) 779.

[35] ERIKSSON, L.-G., MANTSINEN, M., RIMINI, F., et al., Nuclear Fusion 38 (1998) 265.

[36] BILATO, R., BRAMBILlA, M., FENG, Y., and TARDINI, G., p. P5.144, 37th EPS Conference on Plasma Physics, 2010.

[37] WOLFRAM RESEARCH, I., Mathematica, Champaign, Illinois, 2008. 
[38] Rosenbluth, M. N., MACDOnAld, W. M., and JUDD, D. L., Phys. Rev. 107 (1957) 1.

[39] BOSCH, H.-S. and HALE, G., Nuclear Fusion 32 (1992) 611.

[40] MEdLey, S., GORElenKOV, N., ANDRE, R., et al., Nuclear Fusion 44 (2004) 1158.

[41] HERRMANN, A. and GRUBER, O., Fusion Science and Technology 44 (2003) 569.

[42] MEISTER, H., HORTON, L., KURZAN, B., et al., pp. P-1.136, 30th EPS Conference on Plasma Physics, 2003. 